\section{Technikentwicklung in der Pflege aus gerontologischer Perspektive}

\author{
von Andreas Kruse und Eric Schmitt, Institut \\ für Gerontologie, Universität Heidelberg
}

Im vorliegenden Beitrag wird Technikentwicklung aus gerontologischer Perspektive als ein wichtiger Beitrag zur Entwicklung einer altersfreundlichen Kultur behandelt. Im Kontext aktueller Befunde zu Wohnsituation, Versorgungspräferenzen und Technikakzeptanz im Alter werden zunächst Möglichkeiten der Unterstützung selbständiger Lebensführung in Privathaushalten älterer Menschen durch Sensortechnik, Informations- und Kommunikationstechnik sowie Telemedizin aufgezeigt. Die folgenden Abschnitte verdeutlichen die Potenziale von Technikentwicklung für die Betreuung und Versorgung demenzkranker Menschen. Diese liegen zum einen im Aufbau kognitiver Reserven, Frühdiagnostik und Aktivierung, zum anderen in der Förderung von Teilhabe und Lebensqualität, wobei insbesondere Bezogenheit und Selbstgestaltung als zentralen menschlichen Bedürfnissen Rechnung zu tragen ist.

The following article looks at technology development from a gerontological perspective as an important contribution to an age-friendly culture. In the context of current findings regarding living situations, care preferences and technology acceptance of elderly people the possibilities of supporting an independent lifestyle at home through sensor technologies, information and communication technologies as well as tele-medicine are illustrated. The following text shows the potentials of technology development for the care and support of people with dementia. These lie in building up cognitive reserves, early diagnostics and activation, but also in the advancement of participation and quality of life. Especially important is the possibility to shape one's own life, which, as a central human need, should be accounted for.

Die Verfügbarkeit und Nutzung von Technik ermöglicht, erleichtert und unterstützt eine an individuellen Bedürfnissen, Interessen und Neigungen orientierte Lebensführung. Gerade im hohen Lebensalter, wenn die Verletzlichkeit des Menschen spürbar zunimmt und die Möglichkeiten der Aufrechterhaltung eines weitgehend selbständigen, selbst- und mitverantwortlichen Lebens zunehmend von Gegebenheiten der sozialen, räumlichen und infrastrukturellen Umwelt abhängen, kann Technik Selbständigkeit, Kommunikation und Mobilität unterstützen. Zudem kann sie dabei helfen, bereits eingetretene Fähigkeitsdefizite oder allmählich nachlassende Fertigkeiten zu kompensieren. Und schließlich kann sie zu neuen Aktivitäten anregen und neue Optionen der Alltagsgestaltung eröffnen. Aus gerontologischer Perspektive ist Technikentwicklung nicht zuletzt als potenzieller Beitrag zur Entwicklung einer altersfreundlichen Kultur von Interesse: Entsprechend steht Technikentwicklung in der Pflege nicht allein (und auch nicht notwendigerweise primär) unter der Zielsetzung der Kompensation zunehmender Funktionsverluste. Sie zielt vielmehr auch auf die Realisierung von Möglichkeiten zur Selbst- und Weltgestaltung, und dies ganz unabhängig vom Vorliegen unterschiedlicher Beeinträchtigungen (Charness/ Schaie 2003; Kruse 2012).

\section{Technik als Beitrag zur Unterstützung selbständiger Lebensführung in Privathaushalten}

In der Generali Altersstudie 2013 (Institut für Demoskopie Allensbach und Generali Zukunftsfonds 2013), einer repräsentativen Erhebung zur Lebenssituation der 65- bis 85-Jährigen in Deutschland, bezeichneten lediglich 31 Prozent der Befragten ihre Wohnung als altersgerecht, 65 Prozent meinten, diese sei für ältere Menschen nur eingeschränkt geeignet. Von letzteren geben 8 Prozent an, mit ihrer nicht altersgerechten Wohnsituation Probleme zu haben, wobei dieser Anteil erwartungsgemäß deutlich mit dem Lebensalter korreliert: Unter den 65- bis 69-Jährigen liegt dieser bei nur 4 Prozent, unter den 75bis 79-Jährigen bereits bei 9 Prozent und unter den 80- bis 85-Jährigen bei immerhin 14 Prozent.

Als besonders wichtige Maßnahme zur altersgerechten Gestaltung der eigenen Wohnung stufen 65 Prozent ein barrierefreies Badezimmer, 59 Prozent die Möglichkeit, Treppen zu vermei- 
den, 53 Prozent ein Hausnotrufsystem, 34 Prozent eine altersgerechte Küche, 25 Prozent eine elektronische Sicherung der Wohnung, 23 Prozent die Übermittlung medizinischer Daten an den Hausarzt und 20 Prozent eine automatische Erinnerung, wenn Medikamente eingenommen werden müssen, ein. Dabei wäre jeweils der deutlich größere Teil (> 70 Prozent) jener Personen, die eine Maßnahme als besonders wichtig erachten, auch bereit, einen Großteil der anfallenden Kosten selbst zu tragen. Diese Ergebnisse verdeutlichen, dass der Beitrag der Technik zu altersgerechtem Wohnen von den älteren Menschen sehr wohl gesehen wird. In Übereinstimmung mit Befunden anderer Studien ist festzustellen, dass die Einschätzung, ältere Menschen seien technischen Innovationen gegenüber eher reserviert, sie zeigten hier erhebliche Berührungsängste, nicht zutrifft (BMFSFJ 2006; Claßen 2012).

Unter der Voraussetzung, dass ein selbständiges Leben in der eigenen Wohnung alleine nicht mehr möglich ist, würden 59 Prozent ein Leben in der eigenen Wohnung mit Unterstützung durch einen ambulanten Pflegedienst bevorzugen, 31 Prozent würden dann am liebsten in einem Seniorenwohnheim mit eigener Wohnung leben. Ein Leben im Seniorenwohnheim im eigenen Zimmer (21 Prozent), ein Leben bei den eigenen Kindern (20 Prozent) und ein Leben in einer Wohnung in einem Mehrgenerationenhaus (19 Prozent) erscheinen für jeweils etwa ein Fünftel, ein Leben in einer Wohngemeinschaft mit anderen älteren Menschen für 12 Prozent als präferierte Alternative. Dabei zeigten sich in Abhängigkeit von der Schulbildung deutliche Unterschiede in der Präferenz der einzelnen Alternativen, insbesondere mit Blick auf innovative Wohnformen. Unter den Älteren mit hoher Schulbildung liegt etwa der Anteil jener, die ein Leben im Mehrgenerationenhaus als eine von mehreren möglichen Präferenzen angab mit 26 Prozent doppelt so hoch wie jener, die ein Leben bei Kindern oder Enkeln als eine mögliche Präferenz wählten, während unter den Älteren mit einfacher Schulbildung der Anteil jener, der ein Leben bei Kindern oder Enkeln präferiert mit 28 Prozent 2,5 mal höher liegt als der Anteil jener, die das Leben in einem Mehrgenerationenhaus als eine mögliche Präferenz angeben.
Auch wenn das Vorliegen einer (fortgeschrittenen) Demenz den häufigsten Grund für den Umzug in eine stationäre Einrichtung bildet, leben derzeit zwei Drittel der Demenzkranken in Privathaushalten, ein erheblicher Teil auch alleine. Auch bei Vorliegen einer Demenz ziehen es Menschen im Allgemeinen vor, in der eigenen Wohnung bzw. der gewohnten Umgebung zu bleiben. Wie für ältere Menschen generell, so gilt auch für Demenzkranke: (a) Im Vergleich zu jüngeren Menschen wird weit mehr Zeit in der Wohnung verbracht; (b) Freizeitinteressen und Freizeitaktivitäten konzentrieren sich stärker auf den innerhäuslichen Bereich und das unmittelbare Wohnumfeld; (c) subjektiv angemessenes Wohnen ist eine Voraussetzung für die Verwirklichung weiterer zentraler Bedürfnisse, so v. a. dem nach sozialer Teilhabe, nach Aufrechterhaltung des Freundes- und Bekanntenkreises, nach Rückzugsmöglichkeiten und Privatheit, nach Wahrung persönlicher Identität; (d) der Wohnung kommt große Bedeutung für Lebenszufriedenheit und Lebensqualität zu.

Im Falle sensorischer und motorischer Einbußen stellt die Implementierung von Sensorsystemen im vertrauten Wohnumfeld eine bedeutende Maßnahme dar, um Verletzungen zu vermeiden. Diese Sensorsysteme erfassen typische und abweichende Bewegungsmuster (grundlegend dazu schon Mix et al. 2000; s. auch Körtke et al. 2006). Damit wird ein Beitrag zur Vermeidung von Stürzen geleistet, die ihrerseits eine der häufigsten Ursachen für die Einschränkung bzw. den Verlust der Selbständigkeit bilden (Renteln-Kruse/Krause 2004). 30 Prozent der über 65-Jährigen stürzen mindestens einmal pro Jahr, bei über 80-Jährigen steigt diese Zahl auf über 80 Prozent. In mehr als 20 Prozent der Fälle tritt eine Verletzung, zumeist eine Fraktur (fast 60 Prozent der Fälle) auf. Konventionelle Maßnahmen zur Erfassung möglicher Sturzrisiken können dabei nur etwa ein Sechstel der Sturzereignisse verhindern. Entsprechend wird die Notwendigkeit der Implementierung neuer assistierender Technologien zur Überwachung relevanter Bewegungsparameter wie auch zum frühzeitigen Erkennen von Stürzen in der Wohnung betont (Becker/Pfeiffer 2012). 


\section{Informations- und Kommunikations- technik zur Unterstützung selbständiger Lebensführung im Alter}

Im Fünften Altenbericht der Bundesregierung (BMFSFJ 2006) werden fünf Gestaltungsbereiche differenziert, in denen Informations- und Kommunikationstechnik für die Unterstützung zu Hause lebender älterer Menschen genutzt werden kann: (1) Haus-Notruf-Systeme, die zum Serviceruf erweitert werden können, der zur Vermittlung von Kontakten und Dienstleistungen genutzt werden kann; (2) Internetbasierte Informations-, Orientierungs- und Kommunikationsmöglichkeiten, z. B. in Form von E-Mail-Kontakten, Kontaktbörsen oder Chatrooms; (3) Servicezentralen, die unter Nutzung der Videokonferenztechnologie mit älteren Menschen Netzwerke bilden, wobei die angebotenen Leistungen vom seelsorgerischen Gespräch über die Vermittlung von Dienstleistungen bis hin zum virtuellen Kaffeeklatsch reichen können; (4) „Smart Homes“ mit technischen Lösungen, die die Wohn- und Lebensqualität sowie die Sicherheit erhöhen und insbesondere für beeinträchtigte Menschen die Aufrechterhaltung eines selbständigen Lebens erleichtern sollen. Mit Heinze (2012) ist hier anzumerken, dass sich die meisten Angebote nach wie vor zu stark am technisch Machbaren und weniger an den finanziellen Möglichkeiten, Bedürfnissen, Interessen und Präferenzen der Zielgruppe älterer Menschen orientieren. Schließlich werden (5) Monitoringdienste genannt, die es erlauben, relevante medizinische Daten und Indikatoren aktueller Befindlichkeit kontinuierlich zu erfassen und im Bedarfsfall zu vermitteln: $\mathrm{Zu}$ nennen sind hier etwa automatisierte Verfahren der Messung und Überwachung von Vitalparametern, Sturzmelder oder die Beratung und Unterstützung pflegender Angehöriger mithilfe bildbasierter Übertragungstechnik.

Es ist davon auszugehen, dass die heutigen Generationen älterer Menschen im Vergleich zu früheren Generationen neuen Technologien und dem sog. „e-Health“ positiver gegenüberstehen (Heinze 2012). Zudem verfügen deren Wohnungen zunehmend über die informationstechnischen Voraussetzungen für die Entwicklung des „Gesundheitsstandortes Haushalt", der schon in naher Zukunft erheblich an Bedeutung gewinnen wird. Der Patient kann hier den PC oder Fernseher als
Kommunikationsmedium nutzen, mit deren Hilfe er die jeweils interessierenden medizinischen Vitalparameter (z. B. Gewicht, Blutdruck, Herzfrequenz) an ein lokales medizinisches Versorgungszentrum überträgt, von dem er im Gegenzug individuelles Feedback und positive Bestätigung, Anweisungen, Verhaltensregeln und Tipps sowie auf seinen Bedarf abgestimmte Schulungen erhält.

Durch den Einsatz neuer Technologien, die in der Lage sind, für die Diagnosestellung und Behandlung wichtige gesundheitliche Informationen zu erfassen und sicher zu übermitteln, besteht für Patienten die Möglichkeit, ohne großen Aufwand den Kontakt zu betreuenden medizinischen Einrichtungen zu halten, wodurch die Weiterbetreuung nach einer Klinikentlassung erheblich erleichtert wird. Entsprechend birgt eine Ausweitung der Telemedizin auch das Potenzial, sorgende Gemeinschaften (Klie 2014) zu entlasten, zum Teil auch Betreuungs- und Unterstützungsleistungen in sorgenden Gemeinschaften überhaupt erst zu ermöglichen. Für Ärzte ergibt sich die Gelegenheit, Zweitmeinungen einzuholen und damit Diagnosen abzusichern. Auf dem Telemedizin-Portal der Fraunhofer-Gesellschaft finden sich mehr als 200 innovative Projekte, in denen sichere Datenleitungen von Krankenhäusern und niedergelassenen Ärzten für die Übermittlung von Gesundheitsdaten genutzt werden ${ }^{1}$. Im Projekt „CCS Telehealth Ostsachsen“ wurden zur Verdeutlichung der Leistungsfähigkeit einer Telemedizinplattform drei Anwendungen entwickelt: die Etablierung einer Fernanwendung für die Versorgung und Nachsorge von Patienten mit Herzschwäche (Telecoaching), die Organisation der ambulanten Nachsorge von Schlaganfallpatienten durch Case Manager (Tele-Stroke) und die Erfassung von Gewebeschnitten und deren Übermittlung an die Partnerklinik zur Zweitbefundung (Telepathologie). Der vom Bundeskabinett am 27. Mai 2015 verabschiedete e-Health-Gesetzentwurf sieht die flächendeckende Einführung eines modernen Stammdatenmanagements im Anschluss an eine bundesweite Erprobung in Testregionen ab dem 1. Juli 2016 im Zeitraum von zwei Jahren vor. Ab 2018 sollen Notfalldaten auf der Gesundheitskarte gespeichert werden können, wenn der Patient dies wünscht. Ärzte sollen für die Erstellung entsprechender Daten- 
sätze einen Vergütungszuschlag erhalten. Zudem sieht der Gesetzentwurf ab dem 1. April 2017 eine Vergütung von Telekonsilen bei der Befundbeurteilung von Röntgenaufnahmen vor; zur Frage der Erbringung und Vergütung weiterer telemedizinischer Leistungen sollen im Rahmen der Selbstverwaltung Vorschläge erarbeitet werden.

\section{Technik, Frühdiagnostik, kognitive Reserve und Aktivierung}

Neuere Studien zu protektiven Faktoren bei Demenz legen nahe, dass rege geistige Tätigkeit, Bildung, berufliche Fertigkeiten, Sprachvermögen sowie ein aktives Sozialleben zum Aufbau kognitiver Reserven beitragen, die verbesserte Kompensationsmöglichkeiten zur Folge haben (Prince et al. 2012). Auch wenn aktuell weder Präventionsmaßnahmen, die ein Auftreten neurodegenerativer Demenzen verhindern könnten, noch therapeutische Maßnahmen, die die Progredienz der Erkrankung stoppen oder diese heilen könnten, zur Verfügung stehen, ist die kognitive und nichtkognitive Symptomatik durch pharmakologische und psychosoziale Interventionen beeinflussbar (Pantel et al. 2010). Entscheidend ist hier, dass das Vorliegen einer Demenz möglichst frühzeitig erkannt wird. Dies zum einen, um den Betroffenen und ihren Angehörigen zu ermöglichen, sich auf die im Laufe der Erkrankung zunehmenden Verluste und die mit diesen einhergehenden Belastungen und Anforderungen in Teilen einzustellen sowie rechtliche, finanzielle und sonstige Entscheidungen frühzeitig zu treffen (Ding-Greiner 2010; Schröder/Pantel 2011). Studien belegen, dass sowohl pharmakologische als auch psychosoziale Interventionen v. a. in früheren Stadien der Erkrankung positive Auswirkungen haben. Dies gilt insbesondere für Interventionen mit dem Ziel, die kognitive Leistungsfähigkeit zu beeinflussen. An dieser Stelle sei darauf hingewiesen, dass - auch wenn die kognitive Plastizität mit fortschreitendem Alter zurückgeht - Menschen selbst im hohen Alter in der Lage sind, Neues zu lernen, insbesondere auch Strategien zu erwerben, durch die die zunehmenden Alternsverluste in basalen kognitiven Fähigkeiten (in Grenzen) kompensiert werden können. Studien zu den Möglichkeiten der Steigerung kognitiver Leistungsfähigkeit durch kognitives und körperliches Training sprechen dafür, dass Trainingsgewinne bei höherem Ausgangsniveau stärker ausfallen und nachhaltiger sind (Singer et al. 2003). Interventionsstudien sprechen dafür, dass auch Menschen mit einer leichten kognitiven Einbuße (Mild Cognitive Impairment, MCI) erheblich von kognitiven Trainings profitieren können (Hampstead et al. 2012), während etwaige Verbesserungen bei Menschen mit Demenz globalere Maße, wie den allgemeinen neuropsychologischen Status oder die Lebensqualität, nicht beeinflussen (Davis et al. 2001).

Vor dem Hintergrund der Aussagen zu den in verschiedenen Stadien der Erkrankung bestehenden Interventionsmöglichkeiten sei auf Potenziale einer detaillierten Erfassung relevanter Symptome und ihrer Entwicklung verwiesen: dies sowohl im Sinne einer beiläufigen Testung von zentralen Aspekten kognitiver Leistungsfähigkeit wie auch im Kontext der Beobachtung von psychischen Symptomen, Verhaltensauffälligkeiten und Emotionalität. Des Weiteren bietet die Integration von Technik Möglichkeiten körperlicher und kognitiver Aktivierung: dies sowohl im Sinne von Anregungen, vorhandene Fähigkeiten und Fertigkeiten im Kontext von als angenehm empfundenen Tätigkeiten zu nutzen, als auch im Sinne von systematischen Trainings, die in Bezug auf konkrete Inhalte wie auch in Bezug auf formale Aspekte (Zeitpunkt, Dauer, Sequenzierung) an Schwankungen von Befindlichkeit und Leistungsfähigkeit ebenso angepasst werden können wie an individuelle Kompensationsbedarfe und Interessen. Innovationen durch Technik sind sowohl im Bereich der Diagnostik als auch im Bereich der Planung, Umsetzung und Evaluation von Interventionsmaßnahmen erkennbar.

\section{Förderung von Teilhabe und Lebensqualität bei Demenz}

In demokratischen Gesellschaften haben Menschen unabhängig von Art und Ausmaß vorliegender Beeinträchtigungen ein Recht auf Teilhabe. Dieses legitimiert sich vor dem Hintergrund der Erkenntnis, dass die Verwirklichung von Teilhabe - verstanden als Möglichkeit, für die eigenen Bedürfnisse, Interessen und Präferenzen einzutreten, die eigene Entwicklung wie auch die 
Entwicklung von Gesellschaft aktiv mitzugestalten, Verantwortung für sich selbst und andere zu übernehmen - als grundlegendes menschliches Bedürfnis zu verstehen ist.

Auch im sehr hohen Alter, auch unter der Bedingung ausgeprägter Verletzlichkeit lassen sich Menschen von dem Motiv leiten, ihr Leben entsprechend eigener Bedürfnisse, Neigungen, Werte und Ziele zu gestalten (Autopoiesis). Individuelle Reaktionen wie Protest und Anklage, Selbstvorwürfe, abnehmendes Engagement, Antriebsverlust und Niedergeschlagenheit lassen sich nicht selten auf eine befürchtete oder eingetretene Einengung der Selbstgestaltung zurückführen. Gerade bei Demenz werden Verhaltensauffälligkeiten, die durch die erlebte Einengung der Selbstgestaltung bedingt oder mitbedingt sind, vielfach als Verhaltensstörungen (fehl-)interpretiert. Eine genaue Analyse des Erlebens und Verhaltens demenzkranker Menschen zeigt weiterhin, dass die Erfahrung von Bezogenheit in allen Phasen der Demenz entscheidende Bedeutung für das Wohlbefinden besitzt. Für die soziale Umwelt Demenzkranker stellt sich entsprechend die Aufgabe, (a) demenzkranke Menschen nicht aus vertrauten sozialen Kontexten auszuschließen, auch wenn sie zur verbalen Kommunikation nicht mehr in der Lage sind und ihre aktuelle Befindlichkeit und Motivlage nur aus Mimik und Gestik erschlossen werden kann, (b) sich primär an den aktuellen Bedürfnissen und Neigungen wie auch an den Ressourcen eines demenzkranken Menschen $\mathrm{zu}$ orientieren und nicht allein eine pathologische und defizitorientierte Sicht dieses Menschen einzunehmen, (c) nicht über die demenzkranken Menschen zu generalisieren, sondern deren Verschiedenartigkeit genauso zu erkennen wie die Verschiedenartigkeit jener Menschen, bei denen keine Demenz vorliegt, (d) keine Graduierung der Menschenwürde in der Hinsicht vorzunehmen, dass demenzkranke Menschen „weniger“ Menschenwürde besäßen, und die grundsätzliche Unterscheidung zwischen demenzkranken Menschen als psychopathologisch ,auffälligen“ und nicht-demenzkranken Menschen als psychopathologisch „unauffälligen“ zu vermeiden. Zudem sollte (e) bedacht werden, dass die Kommunikation mit demenzkranken Menschen ein hohes Maß an Kontinuität und Zeit erfordert (Überblick in
Kruse 2010). In Pflegeforschung und Pflegepraxis wird seit Jahren hervorgehoben, dass die ethisch fundierte Forderung, wonach der Mensch auch in der letzten Phase seines Lebens die Möglichkeit haben muss, seine Würde zu leben, mit den konkreten Arbeitsbedingungen in Pflegeeinrichtungen vielfach nicht in Übereinstimmung zu bringen ist.

Für pflegende Angehörige, Ehrenamtliche und Pflegekräfte kann aus dem Übersehen der bis in späte Stadien der Erkrankung erhaltenen Erlebens- und Ausdrucksfähigkeit - Resten des Selbst, die sich in Unterstützungskontexten aktualisieren - der Eindruck resultieren, eigene Bemühungen würden von den Erkrankten nicht nur nicht anerkannt, sondern kämen diesem genau betrachtet gar nicht mehr zugute. Dieser Eindruck trägt vielfach dazu bei, dass die noch vorhandene Reziprozität in Beziehungen nicht erkannt, eigenes Handeln als sinnlos erfahren, auf eine den Interessen und Neigungen des Erkrankten entsprechende Gestaltung der Beziehung verzichtet wird (Kruse 2010).

Die Integration von Technik in den Alltag demenzkranker Menschen eröffnet zahlreiche Optionen der Förderung von Bezogenheit und Selbstgestaltung. Der Einsatz entsprechender Technik ist nicht selten eine Voraussetzung dafür, dass dem Wunsch, in der eigenen Wohnung zu verbleiben, entsprochen werden kann. Mit Hilfe von Technik können durch Vergesslichkeit bedingte Gefährdungen (z. B. vergessene Herdplatte) erkannt und vermieden, im Bedarfsfall Bezugspersonen über notwendige Unterstützung informiert werden. Des Weiteren tragen intelligente technische Systeme dazu bei, dass demenzkranke Menschen ihren Alltag weiterhin auf der Grundlage eigener Bedürfnisse und Präferenzen gestalten, indem sie Routineabläufe und wiederkehrende Aufgaben übernehmen, indem sie weiterhin prüfen, ob Vorhaben realisiert wurden, oder indem sie das prospektive Gedächtnis durch Kontrollfragen und Erinnerungen unterstützen. Auf die Bedeutung von Technik im Kontext von körperlicher und kognitiver Aktivierung wurde bereits hingewiesen. An dieser Stelle sei hinzugefügt, dass die Verfügbarkeit von Technik nicht zuletzt auch Möglichkeiten der Alltagsgestaltung eröffnet, so z. B. im Kontext von Spielen, aber auch im Kontext der gedanklichen Beschäftigung mit persönlich wichtigen Personen und Dingen. Im Hinblick auf Bezogenheit 
ist hervorzuheben, dass die Integration von Technik es demenzkranken Menschen ermöglichen kann, selbst Einfluss darauf zu nehmen, zu welchem Zeitpunkt Kontakt mit welchen Menschen aufgenommen werden soll. Zum Teil sind Möglichkeiten der Aufnahme von Kontakt auch an die Nutzung von Technik gebunden, was v. a. dann der Fall ist, wenn Kontaktpersonen nicht im näheren Umfeld verfügbar sind und/oder die Mobilität der Betroffenen stark eingeschränkt ist.

Die vielfältigen Funktionen, die Technik im Kontext von Bemühungen um eine Förderung der Lebensqualität demenzkranker Menschen übernehmen kann, seien abschließend am Beispiel einer Forschungskooperation zwischen dem Karlsruher Institut für Technologie (KIT) und dem Institut für Gerontologie der Universität Heidelberg veranschaulicht. In dem innerhalb der Heidelberg Karlsruhe Research Partnership (HEIKA) geförderten Projekt „Technische Unterstützung zur kognitiven und sozialen Aktivierung von Menschen mit Demenz" wurde als Aktivierungsangebot für die Teilnehmer ambulanter und stationärer Demenzgruppen ein MemorySpiel eingesetzt, das auf einem Tablet-PC mit individualisiertem Material gespielt wird, wobei neben Bildern auch Musik und kurze Videosequenzen als Material verwendet wurden.

In diesem Projekt kommt Technik (a) die Funktion der sensorischen Stimulation zu, einer Form der basalen Stimulation, die sich positiv auf das Wohlbefinden auswirkt. Die Tablet-Anwendung hat (b) Aufforderungscharakter: Sie bietet die Möglichkeit, positiv bewertete Handlungsfolgen zu initiieren. Und sie trägt durch die Aktivierung positiv bewerteter Handlungen zum subjektiven Wohlbefinden bei, nicht zuletzt auch deshalb, weil Möglichkeiten der Gestaltung der eigenen Situation eröffnet werden und so auch die Erfahrung von Selbstwirksamkeit gestützt wird. Die Beschäftigung mit dem Tablet (c) stimuliert die Nutzung kognitiver Fähigkeiten, sie fördert zudem (d) die Repräsentation von vertrauten, persönlich bedeutsamen und positiv bewerteten Personen, Gegenständen und Ereignissen und fördert damit die Tendenz zur Selbstaktualisierung wie auch die Erfahrung von Stimmigkeit. Durch das Spielen in der Gruppe wird (e) die Interaktion mit anderen erleichtert und das Erleben von Bezogenheit ge- fördert; zudem führt das individualisierte Material (f) zum Auftreten spontaner, positiv erlebter Erinnerungen, und schließlich wird (g) durch die Abbildung von vertrauten Räumen das Erlernen von Orientierung in diesen Räumen unterstützt.

Die Ergebnisse der als Kombination von Verhaltensbeobachtung und Interviews mit Betroffenen, Angehörigen und Betreuern realisierten Projektevaluation belegen zum einen, dass auch Menschen mit weiter fortgeschrittenen demenziellen Erkrankungen durchaus in der Lage sind, die entwickelte Anwendung selbständig, ohne umfangreichere Unterstützung durch Pflegefachkräfte, zu nutzen. Sie zeigen zum anderen, dass die Teilnehmerinnen und Teilnehmer ambulanter und stationärer Demenzgruppen unabhängig vom Schweregrad der Erkrankung von der angebotenen Technik profitieren, insofern die Aktivität v. a. positive Auswirkungen auf das emotionale Erleben, das Auftreten von Verhaltensauffälligkeiten und Kontakte zu anderen Angehörigen der Gruppe hat.

\section{Abschluss}

Eine bedeutende Zukunftsaufgabe ist nicht nur in der Weiterentwicklung von Technologien zu sehen, sondern auch in der Entwicklung von gesellschaftlichen Konzepten, die darauf zielen, soziale Ungleichheit im Hinblick auf die Versorgung mit innovativen technischen Produkten abzubauen. Die von Hans Jonas bereits in den 1980er Jahren ausgesprochene Warnung, dass hochwertige technische Produkte, die die Lebensqualität, ja sogar die Mortalität beeinflussen, nur von jenen Menschen erworben werden können, die über ausreichende finanzielle Ressourcen verfügen, sodass diese Ressourcen letzten Endes über die Lebensdauer mitentscheiden (Jonas 1986), sei hier stellvertretend für ethische Mahnungen genannt. Es ist zu fordern, dass auch im Hinblick auf die Ausstattung hilfebedürftiger Menschen mit Informations- und Kommunikationstechnologie soziale Ungleichheit abgebaut wird. Im Grunde sollten alle Haushalte auf diese Technologie zurückgreifen können - und jene Haushalte, die die entsprechenden finanziellen Ressourcen nicht vorhalten können, sollten hinsichtlich der Ausstattung eine Förderung durch staatliche Programme erhalten. 


\section{Anmerkung}

1) http://publica.fraunhofer.de/documents/N-315866. html

\section{Literatur}

Becker, C.; Pfeiffer, K., 2012: Technik in Pflege und Rehabilitation. In: Wahl, H.-W.; Tesch-Römer, C.; Ziegelmann, J.P. (Hg.): Angewandte Gerontologie. Stuttgart, S. 507-512

BMFSFJ - Bundesministerium für Familie, Senioren, Frauen und Jugend, 2006: Fünfter Bericht zur Lage der älteren Generation in der Bundesrepublik Deutschland: Potenziale des Alters in Wirtschaft und Gesellschaft. Der Beitrag älterer Menschen zum Zusammenhalt der Generationen. Berlin

Charness, N.; Schaie, K.W., 2003: Impact of Technology on Successful Aging. New York

Claßen, K., 2012: Technik im Alltag. In: Wahl, H.-W.; Tesch-Römer, C.; Ziegelmann, J.P. (Hg.): Angewandte Gerontologie. Stuttgart, S. 499-506

Davis, R.N.; Massman, P.J.; Doody, R.S., 2001: Cognitive Intervention in Alzheimer Disease: A Randomized Placebo-controlled Study. In: Alzheimer Disease and Associated Disorders 15 (2001), S. 1-9

Ding-Greiner, C., 2010: Demenz vom AlzheimerTyp. Grundlagen und Begleiterkrankungen. In: A. Kruse (Hg.): Lebensqualität bei Demenz? Zum gesellschaftlichen und individuellen Umgang mit einer Grenzsituation im Alter. Heidelberg, S. 281-296

Hampstead, B.M.; Stringer, A.Y.; Stilla, R.F. et al., 2012: Mnemonic Strategy Training Partially Restores Hippocampal Activity in Patients with Mild Cognitive Impairment. In: Hippocampus 22/8 (2012), S. 1652-1658

Heinze, R.G., 2012: Der Paradigmenwandel als Gestaltungsaufgabe: Ambivalenzen der Ökonomisierung und Aktivierung des Alterns. In: Kruse, A.; Rentsch, Th.; Zimmermann, H.-P. (Hg.): Gutes Leben im hohen Alter. Das Altern in seinen Entwicklungsmöglichkeiten und Entwicklungsgrenzen verstehen. Heidelberg, S. 173-204

Institut für Demoskopie Allensbach und Generali Zukunftsfonds, 2013: Generali Altersstudie 2013. Wie ältere Menschen leben, denken und sich engagieren. Frankfurt a. M.

Jonas, H., 1986: Prinzip Verantwortung. Frankfurt a. M. Klie, T., 2014: Wen kümmern die Alten? Auf dem Weg in eine sorgende Gemeinschaft. München

Körtke, H.; Heinze, R.G.; Bockhorst, K. et al., 2006: Telemedizinisch basierte Rehabilitation: Nachhal- tigkeit von Nutzen. In: Deutsches Ärzteblatt 103/4 (2006), S. 2921-2924

Kruse, A., 2010: Menschenbild und Menschenwürde als grundlegende Kategorien der Lebensqualität demenzkranker Menschen. In: Kruse, A. (Hg.): Lebensqualität bei Demenz? Zum gesellschaftlichen und individuellen Umgang mit einer Grenzsituation im Alter. Heidelberg, S. 3-26

Kruse, A., 2012: Die Bedeutung von Informationsund Kommunikationstechnologie für eine Anthropologie des Alters. In: Kruse, A. (Hg.): Kreativität und Medien im Alter. Heidelberg, S. 9-30

Mix, S.; Borchelt, M.; Nieczaj, R. et al., 2000: Telematik in der Geriatrie - Potentiale, Probleme und Anwendungserfahrungen. In: Zeitschrift für Gerontologie und Geriatrie 33 (2011), S. 195-204

Pantel, J.; Haberstroh, J.; Schröder, J., 2010: Psychopharmaka im Altenpflegeheim - Zum Wohle der Bewohner? In: Kruse, A. (Hg.): Lebensqualität bei Demenz. Zum gesellschaftlichen und individuellen Umgang mit einer Grenzsituation im Alter. Heidelberg, S. 317-338

Prince, M.; Acosta, D.; Ferri, C.P. et al., 2012: Dementia Incidence and Mortality in Middle-income Countries, and Associations with Indicators of Cognitive Reserve: A 10/66 Dementia Research Group Population-based Cohort Study. In: The Lancet 380 (2012), S. 50-58

Renteln-Kruse, W. von; Krause T., 2004: Sturzereignisse stationärer geriatrischer Patienten - Ergebnisse einer 3-jährigen prospektiven Erfassung. In: Zeitschrift für Gerontologie und Geriatrie 37 (2004), S. 9-14

Schröder J.; Pantel J., 2011: Die leichte kognitive Beeinträchtigung - Klinik, Diagnostik, Therapie und Prävention im Vorfeld der Alzheimer Demenz. Stuttgart

Singer, T.; Lindenberger, U.; Baltes, P.B., 2003: Plasticity of Memory for New Learning in Very Old Age: A Story of Major Loss? In: Psychology and Aging 18 (2003), S. 306-317

\section{Kontakt}

Prof. Dr. Dr. h.c. Andreas Kruse

Institut für Gerontologie

Ruprecht-Karls Universität Heidelberg

Bergheimer Straße 20, 69115 Heidelberg

E-Mail: andreas.kruse@gero.uni-heidelberg.de 